\title{
HCRP1 inhibits TGF- $\beta$ induced epithelial-mesenchymal transition in hepatocellular carcinoma
}

\author{
WENLIN YANG $^{1 *}$, JI-GANG WANG $^{1,2^{*}}$, JIAWEN XU $^{3}$, DANMEI ZHOU ${ }^{1}$, \\ KEHAN REN ${ }^{1}$, CHENJIAN HOU ${ }^{1}$, LINLIN CHEN ${ }^{4}$ and XIUPING LIU ${ }^{1,4}$ \\ ${ }^{1}$ Department of Pathology, School of Basic Medical Sciences, Fudan University, Shanghai 200032; \\ ${ }^{2}$ Department of Pathology, The Affiliated Hospital of Qingdao University, Qingdao, Shandong 266003; \\ ${ }^{3}$ Department of Pathology, Shangdong Provincial Hospital Affiliated to Shangdong University, Jinan, Shandong 250021; \\ ${ }^{4}$ Department of Pathology, The Fifth Hospital of Shanghai, Fudan University, Shanghai 200240, P.R. China
}

Received September 22, 2016; Accepted February 7, 2017

DOI: $10.3892 /$ ijo.2017.3903

\begin{abstract}
Hepatocellular carcinoma-related protein 1 (HCRP1), also known as human vacuolar protein sorting 37 homologue $\mathrm{A}$ (hVps37A), has not been detected or is significantly downregulated in hepatocellular carcinoma (HCC) tissues. However, information on the regulatory mechanisms of HCRP1 in HCC remains unclear. Here we found that the downregulation of HCRP1 in HepG2 cells (with low invasion capacity) significantly enhanced migration and invasion, whereas HCRP1 upregulation in SMMC-7721 cells (with high invasion capacity) generated the opposite result. Interestingly, the morphology of HepG2 cells significantly changed from an epithelial to mesenchymal phenotype after HCRP1 knockdown. Moreover, we observed a decrease in the expression of epithelial cell markers E-cadherin and $\beta$-catenin, and an increase in the expression of mesenchymal cell markers $\mathrm{N}$-cadherin and vimentin. We also observed that the downregulation of HCRP1 induced epithelial-mesenchymal transition (EMT) through the transforming growth factor- $\beta$ pathway. Together, our findings define a novel function for HCRP1 from the perspective of EMT, which is closely associated with the migration and invasion of HCC cells.
\end{abstract}

\section{Introduction}

Hepatocellular carcinoma (HCC) is one of the most common malignancies occurring around the world $(1,2)$. Although there are various clinical therapeutic strategies for $\mathrm{HCC}$, metastasis

Correspondence to: Professor Xiuping Liu, Department of Pathology, School of Basic Medical Sciences, Fudan University, 138 Yixueyuan Road, Shanghai 200032, P.R. China

E-mail: xpliu1228@fudan.edu.cn

${ }^{*}$ Contributed equally

Key words: hepatocellular carcinoma, hepatocellular carcinomarelated protein $1, \mathrm{hVps} 37 \mathrm{~A}$, TGF- $\beta$, metastasis and recurrence often develop (3). A number of molecules involved in HCC development have been identified (4); however, our understanding of their underlying mechanisms is limited.

The hepatocellular carcinoma-related protein (HCRP1) gene, also known as the human vacuolar protein sorting 37 homologue A (hVps37A) gene, is located on chromosome $8 \mathrm{p} 22$, and is frequently deleted in HCC. HCRP1 was first described as a growth inhibitory protein in HCC cells (5). Thereafter, additional physiologic functions of HCRP1 were explored in other tumors such as ovarian, oral and oropharyngeal, breast, and non-small cell lung cancer (6-10). HCRP1 is a member of the endosomal sorting complex required for transport (ESCRT)-I complex and affects lysosomal sorting of EGFR (11). To date, the regulatory mechanism of HCRP1 in HCC remains unclear.

The role of epithelial-mesenchymal transition (EMT) processes as a central factor governing cancer invasion and metastasis has been established in the last decade and involves the activity of a core set of transcription factors that are activated by signal transduction pathways in neoplastic cells (12). Transforming growth factor- $\beta$ (TGF- $\beta$ ) is a potent pleiotropic cytokine that regulates mammalian development, differentiation, and homeostasis in essentially all cell types and tissues, and plays an important role in the induction of EMT in various cancers (13). Strong inducers of EMT such as TGF- $\beta$ are capable of orchestrating both fibrogenesis and carcinogenesis (14). The aim of the present study was to investigate the role of HCRP1 in HCC and elucidate its underlying mechanisms.

\section{Materials and methods}

Cell culture. Two human HCC cell lines, HepG2 and SMMC7721, were obtained from the Chinese Academy of Sciences (Shanghai, China). The HepG2 cells were cultured in DMEM (cat. no. 11995-065, Gibco, Grand Island, NY, USA) and supplemented with $10 \%$ fetal bovine serum (FBS, cat. no. S1810, Biowest, Nuaillé, France), whereas the SMMC-7721 cells were cultured in RPMI-1640 (cat. no. 11875-093, Gibco) with $10 \%$ FBS; both cell lines were incubated at $37^{\circ} \mathrm{C}$ in 
$5 \% \mathrm{CO}_{2}$ atmosphere. The TGF- $\beta$ receptor kinase inhibitor LY364947 (cat. no. S2805, Selleck) was diluted to $10 \mu \mathrm{M}$ with Opti-MEM $^{\circledR}$ (cat. no. 31985062, Gibco). In addition, 10 cases of paraffin-embedded tissues samples (containing HCC and the corresponding non-tumorous liver tissues) were collected from Huashan Hospital of Fudan University. Ethics approval was obtained from the Clinical Research Ethics Committee of Fudan University.

Immunohistochemistry. Four- $\mu \mathrm{m}$-thick sections were prepared from formalin-fixed, paraffin-embedded blocks, deparaffinized in xylene, and rehydrated using a series of graded ethanol washes. After inhibition of endogenous peroxidase and antigen retrieval (microwave irradiation in $0.01 \mathrm{M}$ citrate buffer at $\mathrm{pH}$ 6.0), the sections were respectively incubated with the primary antibodies anti-HCRP1 (cat. no. A1505, ABclonal, College Park, MD, USA) and anti-E-cadherin (cat. no. 3195, Cell Signaling, Beverly, MA, USA) at $4^{\circ} \mathrm{C}$ overnight, followed by horseradish peroxidase conjugated secondary antibodies (Dako, cat. no. K406511, Glostrup, Denmark). PBS was used as negative control instead of the primary antibody. Slides were then developed for $5 \mathrm{~min}$ with the chromogen, 3,3'-diaminobenzidine (DAB), and counterstained with hematoxylin to distinguish the nucleus from the cytoplasm.

Western blot analysis. Total protein was extracted from cells using a RIPA lysis buffer (cat. no. sc-24948, Santa Cruz, Dallas, TX, USA). Total protein concentration was determined by using a BCA protein assay kit (cat. no. P0012S, Beyotime, Beijing, China). Equal amounts of proteins were separated by SDS-PAGE and electrophoretically transferred to polyvinylidene fluoride membranes (cat. no. IPFL00010, Merck Millipore, Darmstadt, Germany). The membranes were blocked with 5\% milk in Tris- $\mathrm{HCl}$ buffered solution for $2 \mathrm{~h}$, and then subsequently incubated overnight at $4^{\circ} \mathrm{C}$ with the respective primary and secondary antibodies anti-mouse $\lg \mathrm{G}(\mathrm{H}+\mathrm{L})$ (cat. no. SA0001-1, Proteintech) and anti-rabbit $\lg \mathrm{G}$ $(\mathrm{H}+\mathrm{L})$ (cat. no. SA0001-2, Proteintech) at room temperature for $1 \mathrm{~h}$. To confirm equal protein loading, the membranes were incubated with GAPDH (cat. no. G9545, Sigma-Aldrich, St. Louis, MO, USA) or $\beta$-actin (cat. no. 8457S, Cell Signaling) as internal controls. The signals were visualized using an enhanced chemiluminescent substrate and detected by using a FluorChem Q imaging system (Protein Simple, Santa Clara, CA, USA). Images from the western blot assay were quantified using the Quantity One ${ }^{\circledR}$ software (Bio-Rad, Hercules, CA, USA). The expression level was normalized with respect to that of the internal control. The following antibodies were used: anti-HCRP1 (cat. no. A1505, ABclonal), anti-Ecadherin (cat. no. 3195, Cell Signaling, Beverly, MA, USA), anti-N-cadherin (cat. no. 4061, Cell Signaling), anti-vimentin (cat. no. 5741, Cell Signaling), anti-MMP9 (cat. no. 2270, Cell Signaling), anti- $\beta$-catenin (cat. no. 8480, Cell Signaling), anti-Snail (cat. no. 3879, Cell Signaling), Smad2 (phosphoThr220) antibody (cat. no. 11323, SAB, College Park, MD, USA), anti-Smad2 (cat. no. 21322, SAB).

Real-time quantitative PCR ( $q P C R)$. Total RNA was extracted using RNAiso Plus (cat. no. 9108, Takara, Dalian, China) according to the manufacturer's instructions, and then reversed to cDNA using PrimeScript ${ }^{\circledR}$ RT Master Mix (cat. no. DRR036A, Takara) at $37^{\circ} \mathrm{C}$ for $15 \mathrm{~min}, 85^{\circ} \mathrm{C}$ for $5 \mathrm{sec}$, and then at $4^{\circ} \mathrm{C}$. QPCR for HCRP1 and GAPDH was performed in a $10-\mu 1$ reaction volume using the $\mathrm{SYBR}^{\circledR}$ PremixEx $\mathrm{Taq}^{\mathrm{TM}}$ (cat. no. RR420A, Takara) and ABI7900HT real-time PCR system (Life, Singapore). The thermal cycle conditions consisted of one cycle at $95^{\circ} \mathrm{C}$ for $10 \mathrm{~min}$, followed by 40 cycles of amplification at $95^{\circ} \mathrm{C}$ for $15 \mathrm{sec}$, and then $60^{\circ} \mathrm{C}$ for $1 \mathrm{~min}$. The expression level of HCRP1 mRNA was normalized to the geometric mean of the GAPDH mRNA, which was obtained by calculating for the $2^{-\Delta \Delta C t}$ value, whereas $\mathrm{Ct}$ value represented the threshold cycle for each transcript (HCRP1 primers: forward, 5'-CAACAAGUCAUACCACAGCTT-3'; and reverse, 5'-GCUGUGGUAUGACUUGUUGTT-3'. GAPDH primers: forward, 5'-CTGACTTCAACAGCGACACC-3'; and reverse, 5'-TGCTGTAGCCAAATTCGTTGT-3').

Lentiviral-vector infections. Lentiviral vectors expressing a constitutively active form of HCRP1 (HanBio, Shanghai, China) were respectively introduced into SMMC-7721 cells via standard infections at a multiplicity of infection (MOI) of 1:5 for $24 \mathrm{~h}$. To knock down HCRP1 expression, small interfering RNAs (siRNAs) (sequences: 1, 5'-GAAAGUAGCUG CACAUGA AdTdT-3', 5'-UUCAUGUGCAGCUACUUUC dTdT-3'; 2, 5'-CACCAUAAACAACCUGACAdTdT-3', 5'-UGU CAGGUUGUUUAUGGUGdTdT-3'; and 3, 5'-CUCUCAGAA CUAAGUGUGUdTdT-3', 5'-ACACACUUAGUUCUGAGAG dTdT-3') were delivered into the cells. The silencing efficiency was validated by QPCR and the third sequence was selected for recombination with a HCRP1-siRNA lentivirus (shHCRP1) (HanBio) and used in infecting the HepG2 cells. An empty lentiviral vector was used as negative control. The stable clones were selected in media containing puromycin dihydrochloride (cat. no. P9620, Sigma-Aldrich, Shanghai, China) for two weeks.

Wound healing assays. HepG2 and SMMC-7721 cells (density: $5 \times 10^{5}$ cells $/ \mathrm{ml}$ ) were seeded onto 6-well plates in triplicate and incubated overnight. Wound healing assays were performed with a $1,000-\mu 1$ sterile pipette tip, which was used in making a scratch across the confluent monolayer. Marked fields were observed at $0,12,36$ and $48 \mathrm{~h}$ to assess the rate of gap closure. Only the time-points at which the effect was clearly observed were selected. Each assay was performed in triplicate and repeated thrice.

Cell migration and invasion assay. The cell migration study was performed using 24-well tissue culture plates with Transwell ${ }^{\circledR}$ chambers (cat. no. 3422, Corning, NY, USA) according to the manufacturer's instructions. The cell invasion study was performed using a polycarbonate membrane that was precoated with $30 \mu \mathrm{l}$ of Matrige $\mathrm{l}^{\circledR}$ (cat. no. 354234, $\mathrm{BD}$, Shanghai, China) at $37^{\circ} \mathrm{C}$ for $4 \mathrm{~h}$. The cells were detached with trypsin and washed with serum-free medium. HepG2 cells and SMMC-7721 cells (both at densities of $5 \times 10^{4}$ cells $/ \mathrm{ml}$ ) were added to the upper chamber that contained a total volume of $100 \mu \mathrm{l}$ of serum-free medium. The lower chamber contained $600 \mu \mathrm{l}$ of conditioned medium (DMEM with $10 \%$ FBS or RPIM-1640 with $10 \%$ FBS). After $24 \mathrm{~h}$, the cells that adhered to the bottom side of the membrane were fixed in methanol, 

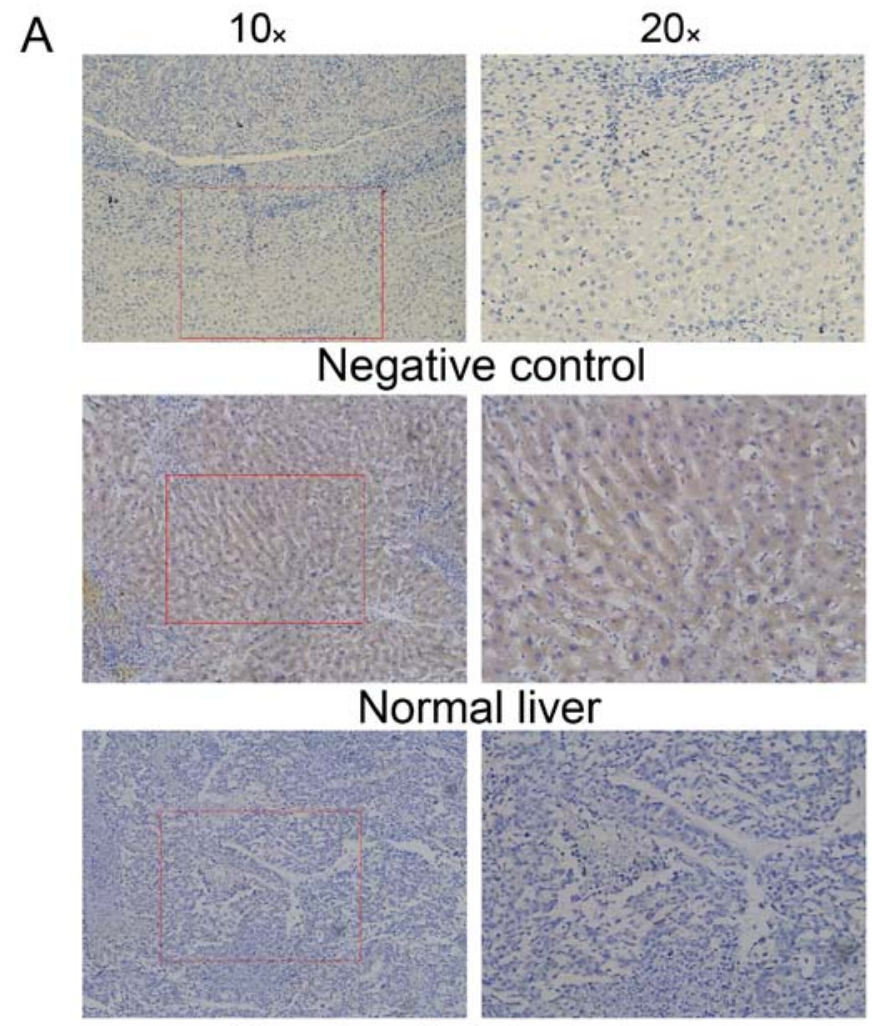

Hepatocellular carcinoma

B

Figure 1. HCRP1 expression in HCC tissues and cells. (A) Immunohistochemical analysis of HCRP1 expression in 10 pairs of HCC tissues and the corresponding adjacent non-cancerous liver tissues. PBS was used as negative control. The results showed that HCRP1 was downregulated in HCC tissues. (B) We also examined the expression of HCRP1 in several HCC cell lines by western blotting. Compared to the normal liver cell line, LO2, HCRP1 was downregulated in the HCC cell lines.

stained with $0.1 \%$ crystal violet, and the average cell number per high power field was calculated. Each assay was conducted in triplicate and repeated thrice.

Statistical analysis. Statistical analysis was performed using Graphpad Prism ${ }^{\circledR} 5.0$ (La Jolla, CA, USA). Numeration data were analyzed using the chi-square test, and the exact probability method was used when there was a theoretical frequency of $<5$. The Student's t-test was used to compare the difference between the two groups. P-values $<0.05$ were considered statistically significant.

\section{Results}

HCRP1 is downregulated in HCC tissues and cell lines. Immunohistochemistry analysis was performed to examine the expression of HCRP1 in tissue samples from 10 cases of HCC. HCRP1 was downregulated in HCC tissues (Fig. 1A).
Then, we assessed the expression of HCRP1 in various HCC cell lines (i.e., HepG2, Hep3B, hun-7, BEL-7404, SMMC-7721, and $\mathrm{MHCC} 97-\mathrm{H}$ ), which was also downregulated compared to that of the normal liver cell line, LO2 (Fig. 1B). The expression of HCRP1 in HepG2 cells (with low invasion capability) was relatively high, whereas that in SMMC-7721 cells (with high invasion capability) was comparatively low. Thus, we performed a knockdown experiment using siRNAs that were transfected into HepG2 cells, as well as functional studies using lentiviral vector-mediated HCRP1-overexpressing SMMC-7721 cells.

HCRPI affects the migration and invasion of HCC cells. The transfection efficiency of the lentiviral vector was validated by RT-PCR and western blot analysis (Fig. 2A), and the biological functions of HCRP1 were also investigated. We performed the wound healing at several time-points $(0,12,24$, 36 and 48 h). Only the time-points at which the effect was 

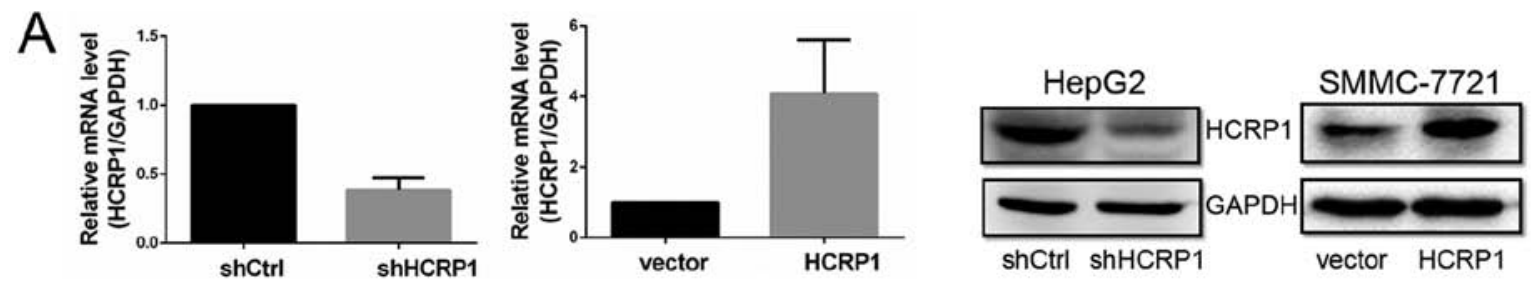

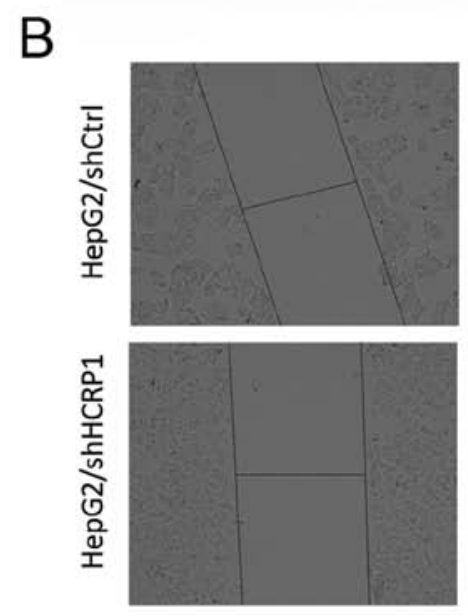

$\mathbf{O h}$

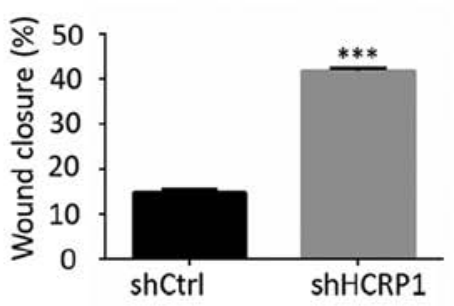

$36 \mathrm{~h}$
C
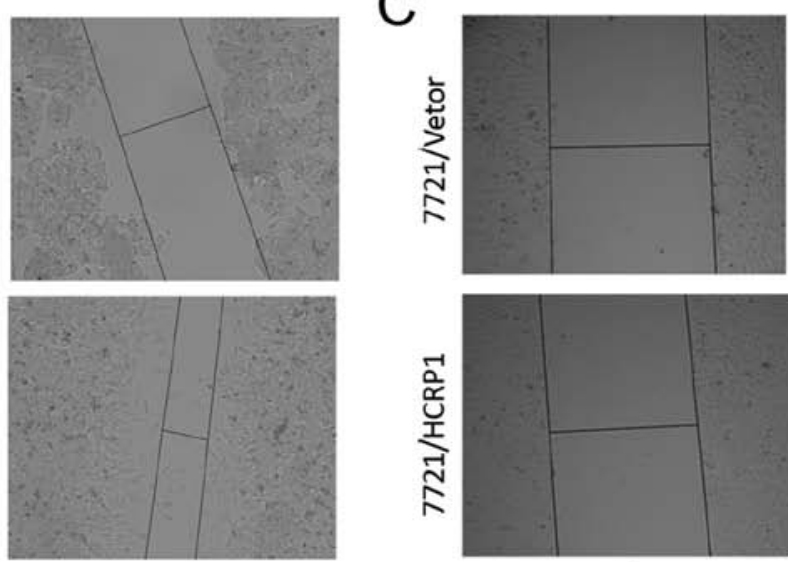

$\mathrm{Oh}$
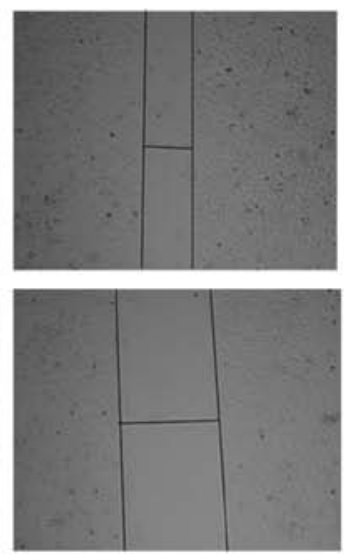

$48 \mathrm{~h}$

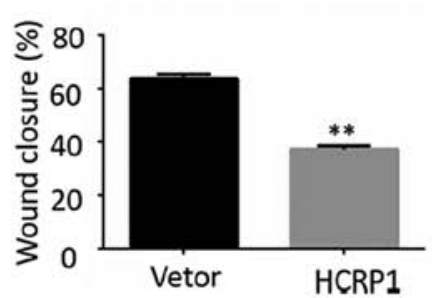

Figure 2. HCRP1 affects the migration of various HCC cell lines. (A) PCR and immunoblotting validation of HCRP1 expression in HepG2/shHCRP1 cells (the best efficiency of HCRP1 deletion cells) and SMMC-7721/HCRP1 cells. (B) An increase in the rate of wound closure in HepG2/shHCRP1 cells was observed. (C) A decrease in the rate of wound closure in SMMC-7721/HCRP1 cells was detected. Each assay was performed in triplicate and repeated thrice. Data are expressed as the mean \pm SEM. ${ }^{*} \mathrm{P}<0.05,{ }^{* *} \mathrm{P}<0.01 ;{ }^{* * *} \mathrm{P}<0.001$.

obviously apparent were selected. Compared to the negative controls, HCRP1-deletion cells (HepG2/shHCRP1 cells) underwent rapid wound closure after $36 \mathrm{~h}$ (Fig. 2B), whereas HCRP1-overexpressing cells (SMMC-7721/HCRP1 cells) exhibited slow wound closure after 48 h (Fig. 2C). Moreover, the Transwell assay showed that knocking down HCRP1 resulted in a significant increase in the migration and invasion of HepG2 cells (Fig. 3A), whereas HCRP1 overexpression decreased the migration and invasion of SMMC-7721 cells (Fig. 3B). Based on these findings, we confirm that HCRP1 inhibits the migration and invasion of HCC cells.

HCRP1 deletion induces HepG2 cells to enter the EMT. The present study observed alterations in the morphology of HepG2/shHCRP1 cells, wherein these became spindleshaped, which is an indication of a mesenchymal phenotype (Fig. 4A). To determine whether HCRP1 is involved in EMT, we investigated a panel of EMT-related markers after HCRP1 knockdown. We observed a significant reduction of E-cadherin expression (epithelial phenotype marker), whereas that of $\mathrm{N}$-cadherin and vimentin (mesenchymal phenotype markers) were upregulated following lentiviral vector-shHCRP1 trans- fection in HepG2 cells (Fig. 4B). We also detected a significant downregulation of $\mathrm{N}$-cadherin and vimentin and an upregulation of E-cadherin in SMMC-7721/HCRP1 cells (Fig. 4B). These results indicated that HCRP1 may be involved in the process of EMT in HCC cells.

HCRPI deletion induces EMT through the TGF- $\beta$ pathway. TGF- $\beta$ is considered as an important inducer of EMT in the progression of various cancers. To investigate whether the TGF- $\beta$ signaling pathway is involved in HCRP1 deletioninduced EMT, we detected the phosphorylation status of Smad2, a downstream effector of the TGF- $\beta$ signaling pathway. We observed a significant increase in the expression of p-Smad2 in HepG2/shHCRP1 cells, which indicated that HCRP1 downregulation also activated the TGF- $\beta$ signaling pathway (Fig. 5A). Furthermore, the expression of MMP9, which is a target of TGF- $\beta$ signaling during EMT, increased after HCRP1 downregulation and decreased after HCRP1 overexpression (Fig. 5B). To further investigate the interaction between HCRP1 and TGF- $\beta$, we used a specific TGF- $\beta$ receptor kinase inhibitor, LY364947, to block the TGF- $\beta$ signaling pathway. The inhibitor counteracted the effects of 


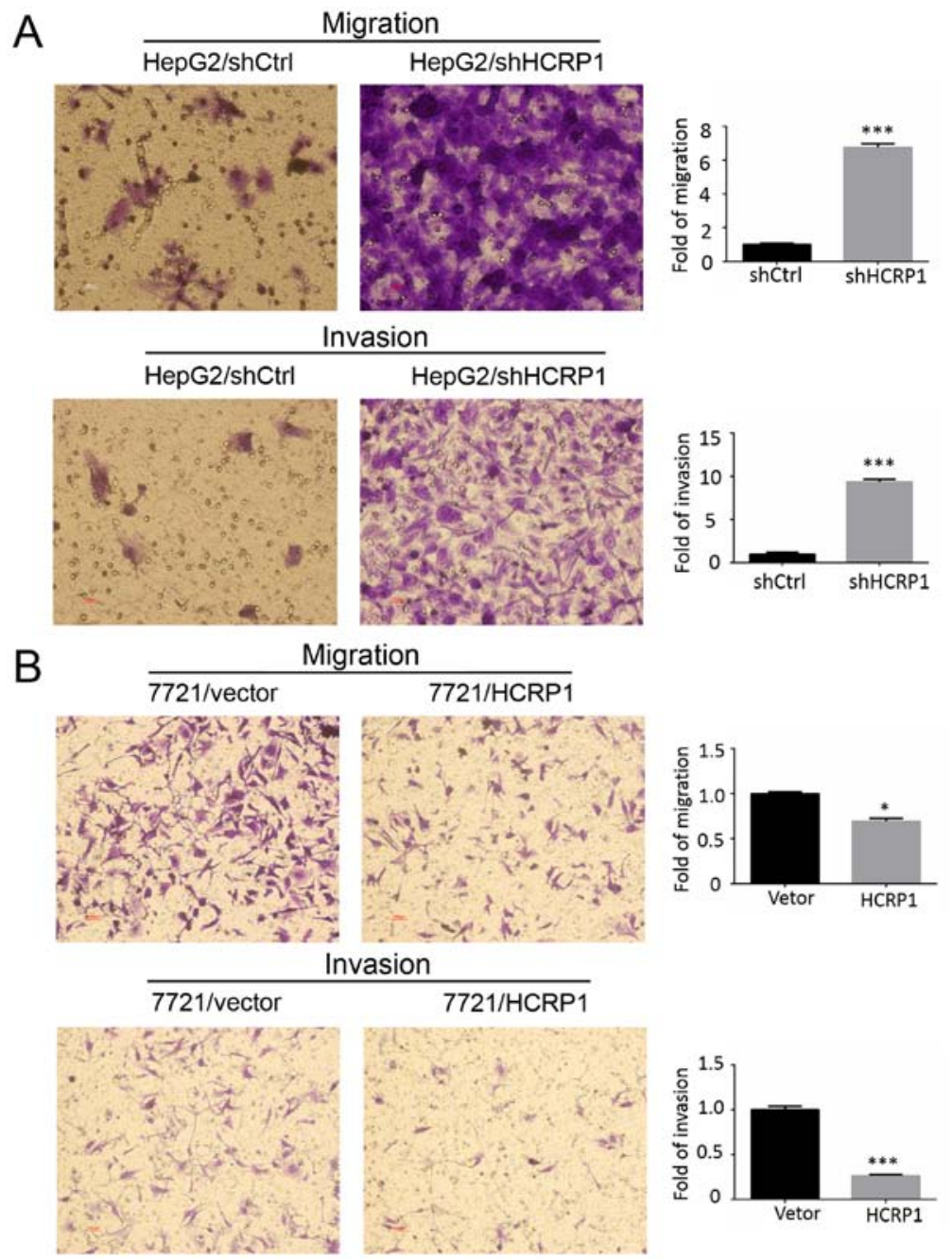

Figure 3. HCRP1 affects migration and invasion in HCC cell lines. (A) The Transwell assays showed that HCRP1 deletion enhanced migration and invasion in HepG2 cells. (B) HCRP1 overexpression decreased the ability for migration and invasion in SMMC-7721 cells. Each assay was performed in triplicate and repeated thrice. Data are expressed as the mean \pm SEM. ${ }^{*} \mathrm{P}<0.05,{ }^{* *} \mathrm{P}<0.01 ;{ }^{* * * *} \mathrm{P}<0.001$.

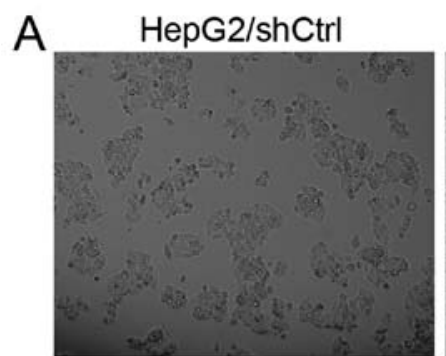

$10 x$

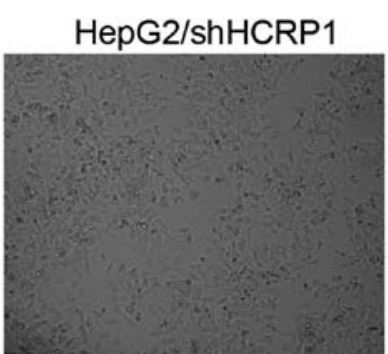

$10 x$

B

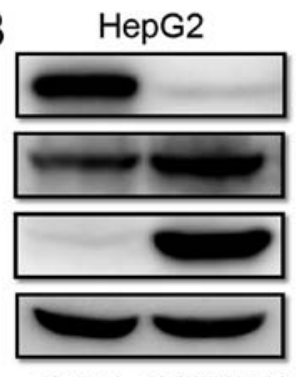

shCtrl shHCRP1
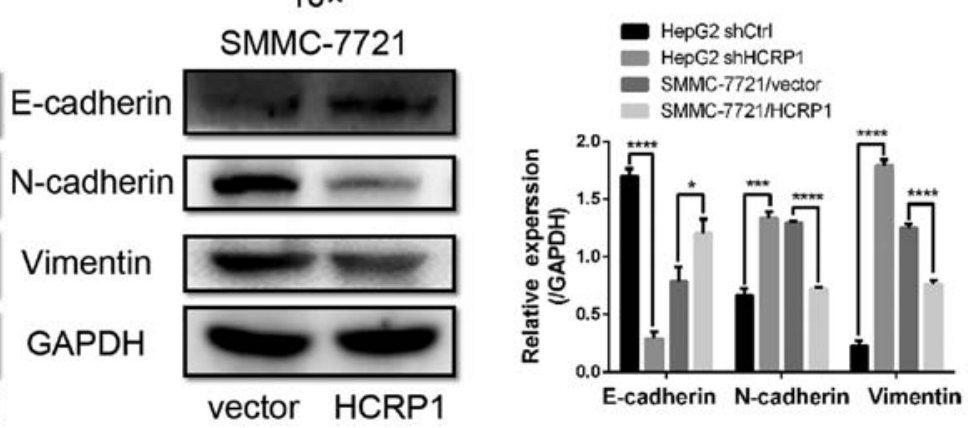

Figure 4. HCRP1 deletion induces EMT in HepG2 cells. (A) The morphology of the HepG2/shHCRP1 cells significantly changed from that of the epithelial phenotype to a mesenchymal phenotype. (B) Immunoblot analysis of the expression of the epithelial marker E-cadherin and the mesenchymal markers $\mathrm{N}$-cadherin and vimentin in HepG2 cells and SMMC-7721 cells. The bar chart refers to semiquantitative analysis of western blotting. Each assay was performed in triplicate and repeated thrice. Data are expressed as the mean $\pm \mathrm{SEM} .{ }^{*} \mathrm{P}<0.05,{ }^{* *} \mathrm{P}<0.01 ;{ }^{* * *} \mathrm{P}<0.001$. 
A
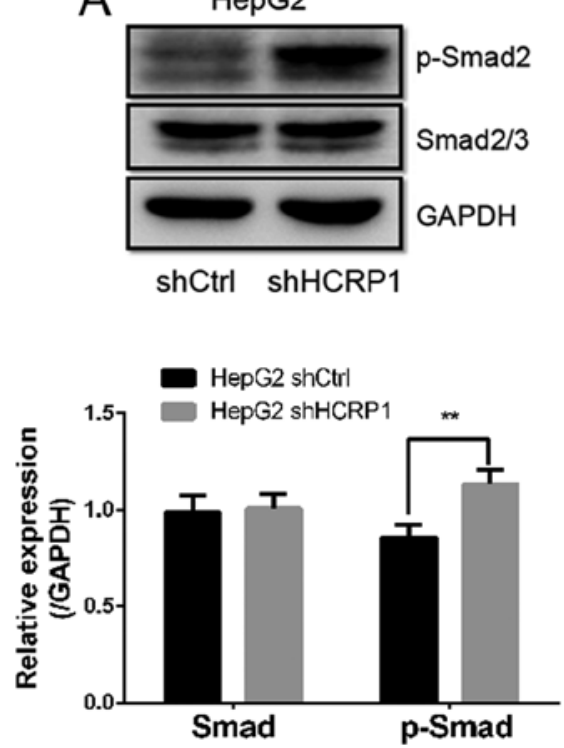

B

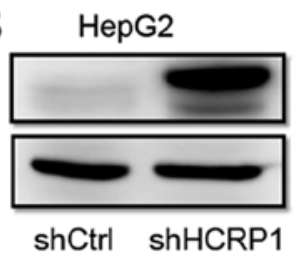

SMMC-7721

MMP9

GAPDH

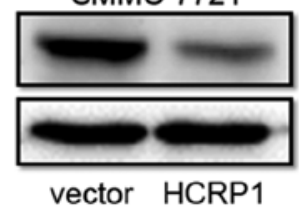

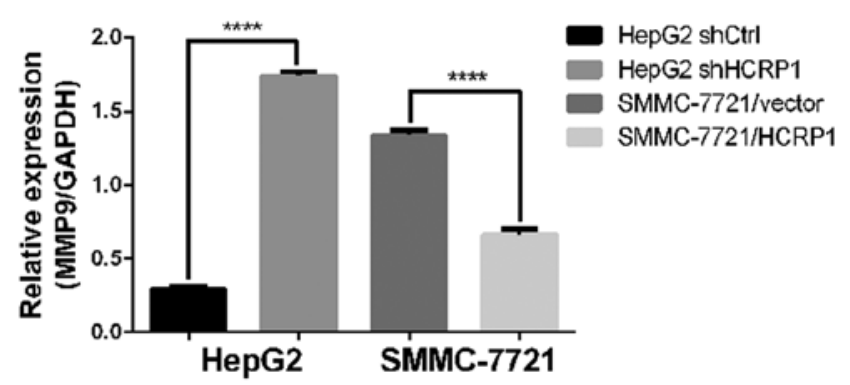

Figure 5. HCRP1 deletion induces EMT via the TGF- $\beta$ pathway. (A) Immunoblots of p-Smad2 and total Smad2/3 protein levels in HepG2/shHCRP1 cells and HepG2/shCtrl cells. (B) MMP9 expression increased in HepG2/shHCRP1 cells and decreased in 7721/HCRP1 cells. Each assay was performed in triplicate and repeated thrice. Data are expressed as the mean \pm SEM. ${ }^{*} \mathrm{P}<0.05,{ }^{* *} \mathrm{P}<0.01 ;{ }^{* * *} \mathrm{P}<0.001$.
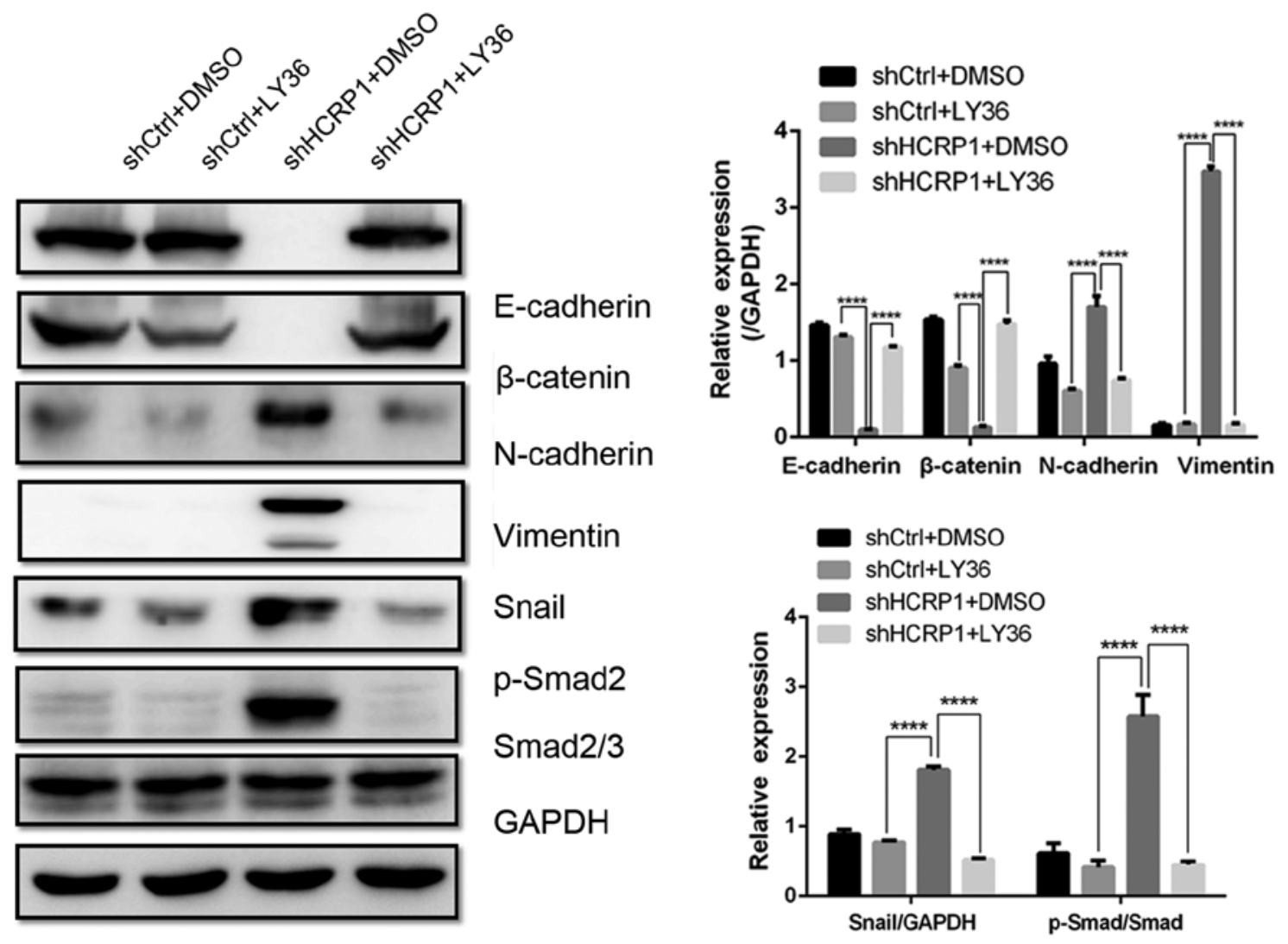

Figure 6. Drug treatment in HCRP1 deletion cells. HepG2/shHCRP1 and HepG2/shCtrl cells were treated with $10 \mu$ M of LY364947 (LY36) and DMSO as control for $48 \mathrm{~h}$. Immunoblot of E-cadherin, $\beta$-catenin, $\mathrm{N}$-cadherin, vimentin, Snail, p-Smad2, and total Smad2/3 protein. Each assay was performed in triplicate and repeated thrice. Data are expressed as the mean $\pm \mathrm{SEM} .{ }^{*} \mathrm{P}<0.05,{ }^{* *} \mathrm{P}<0.01 ;{ }^{* * * *} \mathrm{P}<0.001$.

HCRP1 deletion in HepG2 cells, as indicated by a decrease in the expression of $\mathrm{p}$-Smad2 and mesenchymal cell markers $\mathrm{N}$-cadherin and vimentin, as well as the transcription factor
Snail. In addition, the inhibitor restored the expressions of epithelial cell markers E-cadherin and $\beta$-catenin, which were diminished by HCRP1 deletion (Fig. 6). 

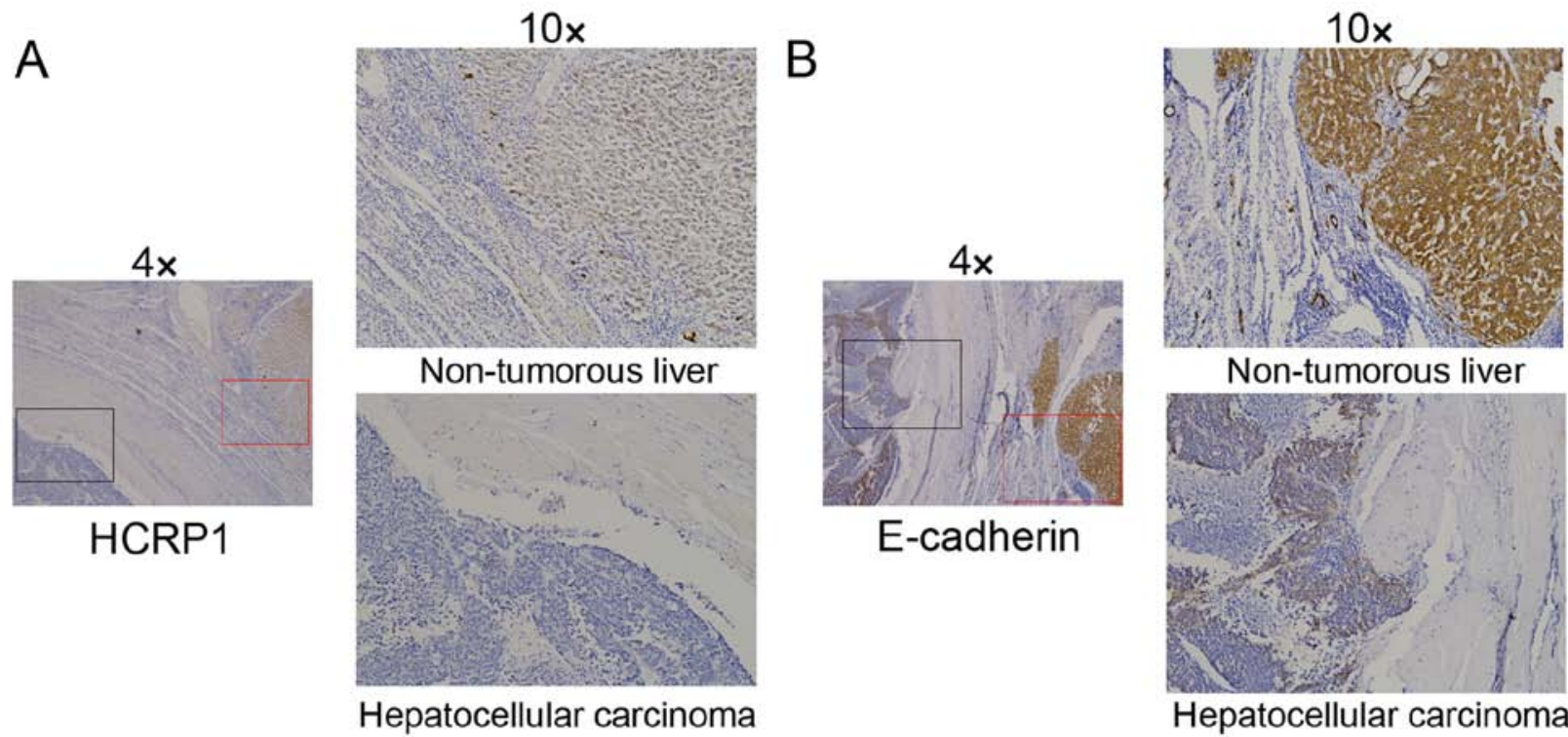

Figure 7. The relationship between HCRP1 and E-cadherin in HCC tissues. (A) Immunohistochemical analysis was performed to detect the expression of HCRP1 and E-cadherin in tissues from 10 cases of HCC tissues. Images are representative traces of one sample and that the tissue specimens were paired. Immunohistochemistry revealed that HCRP1 was abundant in non-tumorous liver tissues and not detected in HCC tissues. (B) E-cadherin was abundant in non-tumorous liver tissues and reduced in expression in HCC tissues.

The relationship between HCRPI and EMT markers in HCC tissues. To further explore the relationship between HCRP1 and EMT, immunohistochemical analysis was performed to detect the expression of HCRP1 and E-cadherin in tissues from 10 cases of HCC tissues. The results revealed that HCRP1 and E-cadherin were not expressed in HCC tissues, whereas highly expressed in non-tumorous liver tissues (Fig. 7).

\section{Discussion}

HCRP1 is a novel HCC-related protein whose gene is located at a frequently deleted region such as a loss of heterozygosity (LOH) region in HCC (4). However, HCRP1 as a novel tumor suppressor gene in $\mathrm{HCC}$, and its biological functions and underlying mechanisms remain elusive. In the present study, we first examined the expression of HCRP1 in 10 pairs of HCC tissues and the corresponding non-cancerous tissues, which showed that HCRP1 was downregulated in HCC tissues. Then, we examined the expression level of HCRP1 in LO2 normal liver cells and various other HCC cell lines, which revealed that HCRP1 was also downregulated in HCC cell lines. In addition, loss/gain-of-function studies (HCRP1 deletion in HepG2 cells and HCRP1 overexpression in SMMC-7721 cells by lentiviral vector infections) showed that HCRP1 is a regulator of cell migration and invasion. Moreover, we demonstrated that HCRP1 downregulation in HCC contributes to EMT, and TGF- $\beta$ participates in this process. Our study provides a novel mechanism underlying HCC metastasis.

The wound healing assay and Transwell assay demonstrated that HCRP1 deletion enhanced the ability for migration and invasion in HepG2 cells, whereas overexpression of HCRP1 inhibited these two activities in SMMC-7721 cells. These results confirmed the role of HCRP1 on migration and invasion in HCC cells. Interestingly, HCRP1 knockdown induced an alteration in cell morphology, which indicated that HCRP1 is involved in the EMT of HCC cells.

EMT plays a crucial role in the early steps of metastasis in cancer progression, including HCC (15). During EMT development, epithelial cells lose their polarity and acquire a mesenchymal phenotype, thereby becoming migratory and invasive (16). The pivotal role of EMT in HCC has been increasingly recognized and various molecular mechanisms underlying hepatocellular EMT have been identified such as TGF- $\beta$ signaling (17). TGF- $\beta$ signaling commences by its binding to three high-affinity receptors, namely, T $\beta$ R-I, T $\beta$ R-II, T $\beta$ R-III, and stimulates the latent transcription factors, $\operatorname{Smad} 2$ and Smad3 (13). Changes in cell behavior regulated by the activation of Smad2/3 are referred to as 'canonical TGF- $\beta$ signaling'. Therefore, to investigate whether HCRP1 deletion induces EMT via the TGF- $\beta$ signaling pathway, we detected the protein expression level of phosphorylated Smad2. We observed a significant increase in the expression of p-Smad 2 in HepG2/shHCRP1 cells. We also detected the expression of MMP9, which is well established as a functional target of TGF- $\beta$ signaling during EMT (18). In addition, we also determined that the TGF- $\beta$ receptor kinase inhibitor LY364947 decreased the expression of p-Smad2 and mesenchymal cell markers N-cadherin and vimentin, as well as the transcription factor Snail, whose levels of expression increased due to the downregulation of HCRP1. All this evidence support that the TGF- $\beta$ pathway is activated during HCRP1 deletion-induced EMT.

The expression of HCRP1 is a predictor of disease-free survival in HCC and could be a helpful predictor that might influence medical decisions in treating HCC (19). A more recent report found that the TGF- $\beta$ inhibitor LY2157299 appears to modulate EMT and has a clinically meaningful benefit to patients (20). Given that TGF- $\beta$ is a downstream signal of HCRP1, we consider TGF- $\beta$ inhibitor as an effective reagent for HCC patients with low HCRP1 expression. 


\section{Acknowledgements}

This study was supported by The National Natural Science Foundation of China (grant nos. 81272387, 81470857 and 81502272). The authors would like to thank LetPub (www. letpub.com) for its linguistic assistance during the preparation of this manuscript.

\section{References}

1. Torre LA, Bray F, Siegel RL, Ferlay J, Lortet-Tieulent J and Jemal A: Global cancer statistics, 2012. CA Cancer J Clin 65: 87-108, 2015.

2. Zhao P, Wang JG, Gao P, Li X and Brewer R: Sudden unexpected death from natural diseases: Fifteen years' experience with 484 cases in Seychelles. J Forensic Leg Med 37: 33-38, 2016.

3. Meguro M, Mizuguchi T, Kawamoto $M$ and Hirata K: The molecular pathogenesis and clinical implications of hepatocellular carcinoma. Int J Hepatol 2011: 818672, 2011.

4. Thorgeirsson SS and Grisham JW: Molecular pathogenesis of human hepatocellular carcinoma. Nat Genet 31: 339-346, 2002.

5. Xu Z, Liang L, Wang H, Li T and Zhao M: HCRP1, a novel gene that is downregulated in hepatocellular carcinoma, encodes a growth-inhibitory protein. Biochem Biophys Res Commun 311: 1057-1066, 2003.

6. Wittinger M, Vanhara P, El-Gazzar A, Savarese-Brenner B, Pils D, Anees M, Grunt TW, Sibilia M, Holcmann M, Horvat R, et al: hVps37A Status affects prognosis and cetuximab sensitivity in ovarian cancer. Clin Cancer Res 17: 7816-7827, 2011.

7. Perisanidis C, Savarese-Brenner B, Würger T, Wrba F, Huynh A, Schopper C, Kornek G, Selzer E, Ewers R, Psyrri A, et al: HCRP1 expression status is a significant prognostic marker in oral and oropharyngeal cancer. Oral Dis 19: 206-211, 2013.

8. Xu J, Yang W, Wang Q, Zhang Q, Li X, Lin X, Liu X and Qin Y: Decreased HCRP1 expression is associated with poor prognosis in breast cancer patients. Int J Clin Exp Pathol 7: 7915-7922, 2014.

9. Yang W, Wang JG, Wang Q, Qin Y, Lin X, Zhou D, Ren K, Hou C, Xu J and Liu X: Decreased HCRP1 promotes breast cancer metastasis by enhancing EGFR phosphorylation. Biochem Biophys Res Commun 477: 222-228, 2016.
10. Du Y, Wang P, Sun H, Yang J, Lang X, Wang Z, Zang S, Chen L, Ma $J$ and Sun D: HCRP1 is downregulated in non-small cell lung cancer and regulates proliferation, invasion, and drug resistance. Tumour 37: 15893-15901, 2016.

11. Bache KG, Slagsvold T, Cabezas A, Rosendal KR, Raiborg C and Stenmark H: The growth-regulatory protein HCRP1/hVps37A is a subunit of mammalian ESCRT-I and mediates receptor downregulation. Mol Biol Cell 15: 4337-4346, 2004.

12. Voutsadakis IA: Epithelial-mesenchymal transition (EMT) and regulation of EMT factors by steroid nuclear receptors in breast cancer: A review and in silico investigation. J Clin Med 5: E11, 2016.

13. Wendt MK, Tian M and Schiemann WP: Deconstructing the mechanisms and consequences of TGF- $\beta$-induced EMT during cancer progression. Cell Tissue Res 347: 85-101, 2012.

14. Giannelli G, Koudelkova P, Dituri F and Mikulits W: Role of epithelial to mesenchymal transition in hepatocellular carcinoma. J Hepatol 65: 798-808, 2016.

15. van Zijl F, Mall S, Machat G, Pirker C, Zeillinger R, Weinhaeusel A, Bilban M, Berger W and Mikulits W: A human model of epithelial to mesenchymal transition to monitor drug efficacy in hepatocellular carcinoma progression. Mol Cancer Ther 10: 850-860, 2011.

16. Kalluri R and Weinberg RA: The basics of epithelial-mesenchymal transition. J Clin Invest 119: 1420-1428, 2009.

17. van Zijl F, Zulehner G, Petz M, Schneller D, Kornauth C, Hau M, Machat G, Grubinger M, Huber $\mathrm{H}$ and Mikulits W: Epithelialmesenchymal transition in hepatocellular carcinoma. Future Oncol 5: 1169-1179, 2009.

18. Duivenvoorden WC, Hirte HW and Singh G: Transforming growth factor betal acts as an inducer of matrix metalloproteinase expression and activity in human bone-metastasizing cancer cells. Clin Exp Metastasis 17: 27-34, 1999.

19. Lai MW, Huang SF, Lin SM, Chen TC, Lin CY, Yeh CN, Yeh TS, Chen MF and Yeh CT: Expression of the HCRP1 mRNA in $\mathrm{HCC}$ as an independent predictor of disease-free survival after surgical resection. Hepatol Res 39: 164-176, 2009.

20. Giannelli G, Villa $E$ and Lahn M: Transforming growth factor- $\beta$ as a therapeutic target in hepatocellular carcinoma. Cancer Res 74: $1890-1894,2014$ 